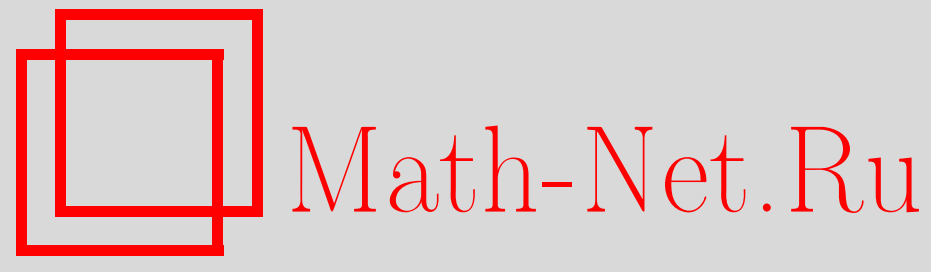

М. В. Шершнева, Метод расчёта ресурса стержневых конструкций на основе энергетического варианта ползучести и длительной прочности, Вестн. Сам. гос. техн. ун-та. Сер. Физ.-мат. науки, 2012, выпуск 1(), 141-149

DOI: https://doi.org/10.14498/vsgtu1038

Использование Общероссийского математического портала Math-Net.Ru подразумевает, что вы прочитали и согласны с пользовательским соглашением

http: //www.mathnet.ru/rus/agreement

Параметры загрузки:

IP : 3.93.64.190

26 апреля 2023 г., $17: 24: 35$

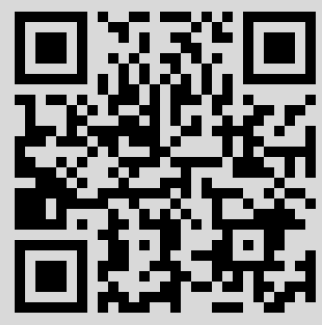


Вестн. Сам. гос. техн. ун-та. Сер. Физ.-мат. науки. 2012.№ 1 (26). С. 141-149

УДК 539.376+539.434

\title{
МЕТОД РАСЧЁТА РЕСУРСА СТЕРЖНЕВЫХ КОНСТРУКЦИЙ НА ОСНОВЕ ЭНЕРГЕТИЧЕСКОГО ВАРИАНТА ПОЛЗУЧЕСТИ И ДЛИТЕЛЬНОЙ ПРОЧНОСТИ
}

\author{
М. В. Шеринева \\ Самарский государственный технический университет, \\ 443100, Россия, Самара, ул. Молодогвардейская, 244. \\ E-mail: mary-sofya@mail.ru
}

\begin{abstract}
Предложен аналитический метод оценки ресурса стержневых элементов конструкиий в условиях ползучести по дебормаиионному критерию отказа. Виполнена линеаризаиия стохастических реологических уравнений энергетического типа, получены оченки времени безотказной работы при ограничениях на пределъные значения деформации ползучести. Выполнена проверка адекватности метода эксперименталъным данным по ползучести образиов из стали $12 X 18$ H10T при $T=850^{\circ} \mathrm{C}$. Наблюдается соответствие расчётных и экспериментальных данных
\end{abstract}

Ключевые слова: ползучесть, предел длительной прочности, стохастическая модель, вероятность безотказной работы, деформационный критерий отказа.

Введение. Подходы к оценке надёжности элементов конструкций в условиях ползучести существенно отличаются от аналогичных задач в условиях склерономного деформирования, поскольку приходится считаться не только с процессом разрушения, но и с предельно допустимыми значениями необратимой реологической деформации, развиваемой во времени. Второй важный факт состоит в значительном разбросе деформации ползучести, который может составлять 50-70\%, и такие данные приходится считать удовлетворительными [1]. Существенное влияние случайных возмущений механических характеристик на поля напряжений и деформаций и необходимость построения соответствующих стохастических моделей для расчётов на прочность отмечались во многих работах (см., например, [1-10]). Широко используемые для оценки ресурса безопасной эксплуатации элементов конструкции феноменологические теории (в частности, и теория ползучести), как правило, носят детерминированный характер и не учитывают явления разброса для тех или иных механических характеристик (деформация ползучести, перемещение, время разрушения и т. д.). Поэтому детерминированный метод расчёта является первым, и в ряде случаев недостаточным, приближением. Неточности детерминированного расчёта на прочность покрываются, например, назначением коэффициента запаса прочности, который во многих случаях выбирается без достаточных оснований и не является оптимальным. Это приводит либо к появлению неиспользуемых резервов прочности элементов конструкций, либо к преждевременному их разрушению. Особенно остро эта проблема стоит для элементов конструкций, эксплуатирующихся в условиях ползучести. Сдерживающими факторами решения задач надёжности являются, во-первых, слабо развитая теория стохастического реологического деформирования, особенно на стадии ускоренной ползучести, а во-вторых -

Мария Викторовна Шершнева, аспирант, каф. прикладной математики и информатики. 
физическая и стохастическая нелинейности определяющих уравнений ползучести и длительной прочности, что не позволяет в должной мере развивать аналитические методы решения стохастических краевых задач. Здесь имеются решения лишь на стадии установившейся ползучести [11-13], и единичные работы посвящены решению краевых задач с учётом третьей стадии ползучести [14]. Широко используемый в задачах ползучести метод МонтеКарло [4, 15-19] требует большой трудоёмкости и представительной выборки экспериментальных данных по ползучести материала, получение которых в необходимом объёме представляет определённые трудности. В отличие от метода Монте-Карло аналитические подходы позволяют широко применять разработанный В.В. Болотиным аппарат оценки надёжности через вероятность безотказной работы [2]. Применительно к стержневым системам в [20] предложен метод оценки надёжности конструкций из стохастически неоднородного материала в условиях первой и второй стадий ползучести по деформационному критерию отказа, а в работе [21] сделана попытка обобщения данного подхода для оценки надёжности с учётом третьей стадии на основании кинетических уравнений Ю.Н. Работнова (но без учёта первой стадии ползучести). Целью настоящей работы является обобщение указанного подхода оценки надёжности стержневых конструкций на основе энергетического варианта реологического деформирования и разрушения материалов [22, 23] на примере стохастически неоднородного стержня, работающего на растяжение (сжатие).

1. Построение и линеаризация стохастической модели. Для описания случайных свойств растягиваемого стержня рассматривается стохастическая модель ползучести [22,23], описывающая вторую и третью стадии, вида

$$
\begin{gathered}
\dot{p}(t)=c \sigma^{n}(t), \quad \sigma(t)=\sigma_{0}(t)(1+\omega(t)), \quad \dot{\omega}(t)=\alpha \sigma(t) \dot{p}(t) ; \\
\omega(0)=0, \quad p(0)=0,
\end{gathered}
$$

где $p(t)$ - деформация ползучести, $\sigma(t)$ и $\sigma_{0}(t)$ - истинное и номинальное напряжения, $c$ и $\alpha$-случайные величины, $n$-детерминированный параметр, $\omega(t)$ - параметр повреждённости.

Решение системы (1) с условиями (2) при $\sigma_{0}(t)=\sigma_{0}=$ const имеет вид

$$
p(t)=-\frac{1}{n \alpha \sigma_{0}} \ln \left|1-n \sigma_{0}^{n+1} \alpha c t\right| .
$$

Уравнение (3) является стохастически нелинейным, следовательно, его нельзя использовать в существующей методике оценки надёжности для стохастических линейных моделей, предложенной в [20]. Поэтому необходимо выполнить линеаризацию (3). Формальное разложение в ряд Тейлора неудобно, так как логарифмический ряд сходится крайне медленно и в соответствующем ряде для достижения заданной точности необходимо удерживать большое количество членов с последующей стохастической оценкой каждого члена при степенях $t$. Поэтому в настоящей работе используется метод аппроксимации функции $\ln (1-x)$ степенными полиномами с использованием интегрального метода наименьших квадратов (ИМНК). При этом рассматривались три варианта аппроксимации:

$$
\ln (1-t) \cong-x+a_{1} x^{2}+a_{2} x^{3}+a_{3} x^{4}
$$




$$
\begin{aligned}
& \ln (1-x) \cong-x+a_{1} x^{2}+a_{2} x^{3} \\
& \ln (1-x) \cong-x+a_{1} x^{2}+a_{2} x^{4}
\end{aligned}
$$

а критерий минимизации брался в виде

$$
\delta=\int_{0}^{0,95}\left(\frac{P_{\text {точн }}(x)-P_{\text {апп }}(x)}{P_{\text {точн }}(x)}\right)^{2} d x \rightarrow \min .
$$

В (7) верхний предел интегрирования $x=0,95$, так как при $x=1$ имеет место асимптотическое поведение функции $y=\ln (1-x) ; P_{\text {точн }}$ и $P_{\text {апп }}-$ соответственно точные значения функций $y=\ln (1-x)$ и одной из функций, стоящих в правой части (4)-(6).

Для нахождения оптимальных значений коэффициентов $a_{i}$ вычисляются частные производные по коэффициентам $a_{i}$ от величины $\delta$ и приравниваются к нулю. Из полученной системы линейных уравнений находятся $a_{i}$ для заданной модели.

В результате для аппроксимаций (4)-(6) соответственно получены следующие значения коэффициентов:

$$
\begin{gathered}
a_{1}=-0,91048, \quad a_{2}=1,90612, \quad a_{3}=-3,06057 \\
a_{1}=-0,0169, \quad a_{2}=-1,6281 \\
a_{1}=-0,1884, \quad a_{2}=-1,9925
\end{gathered}
$$

Проведённые оценки точности аппроксимирующих функций по величине $\delta$ показали, что наибольшая точность достигается при аппроксимации вида (4). Величина $\delta$ в этом случае равна 0,0005 , тогда как для (5) она составляет 0,0022, а для (6) - 0,0021.

Следует отметить, что при величине $\delta=5 \cdot 10^{-4}$ количество членов формального разложения в ряд Тейлора для функции $y=\ln (1-x)$ равно двенадцати, в то время как в аппроксимации (4) - всего трём. Таким образом, функцию (3) в дальнейшем будем аппроксимировать по формуле (4) с коэффициентами, задаваемыми (8). С учётом (3) она будет представлена в следующем виде:

$$
\begin{aligned}
p(t)=c \sigma_{0}^{n} t+0,911 c^{2} \alpha n \sigma_{0}^{2 n+1} & t^{2}- \\
& -1,906 c^{3} \alpha^{2} n^{2} \sigma_{0}^{3 n+2} t^{3}+3,061 c^{4} \alpha^{3} n^{3} \sigma_{0}^{4 n+3} t^{4} .
\end{aligned}
$$

\section{2. Оценка надёжности стержневых конструкций по деформационному кри-} терию отказа. Рассмотрим теперь вероятностные методы оценки прочностной надёжности по деформационным критериям отказов элементов конструкций. Основной количественной характеристикой надёжности является вероятность безотказной работы. Она в данном случае определяет вероятность того, что во всех точках материала элемента конструкции выполняется условие прочности:

$$
p(t) \leqslant p^{*}
$$

где $p^{*}$ - назначенный ресурс по предельно-допустимой накопительной деформации. 
Функция надёжности $P(t)$, описывающая вероятность безотказной работы на отрезке $[0, t]$, равна вероятности пребывания случайной функции $p(t)$ в допустимой области $\left(0, p^{*}\right)$ на этом отрезке времени [2]:

$$
P(t)=P\left\{p(\tau) \in\left(0, p^{*}\right), \tau \in[0, t]\right\} .
$$

В связи с тем, что согласно модели (1), (2) деформация ползучести является неубывающей функцией, функция $p(t)$, покинув в некоторый момент времени область $\left(0, p^{*}\right)$, затем в эту область возвратиться не может. Поэтому для вероятности безотказной работы $P(t)$ на отрезке времени $[0, t]$ имеет место более простая формула [2]:

$$
P(t)=P\left\{p(t) \in\left(0, p^{*}\right)\right\} .
$$

В отличие от общего случая (10), когда вычисление случайной функции требует рассмотрения выбросов случайного процесса, здесь достаточно вычислить вероятность нахождения случайной функции $p(t)$ в заданной области в рассматриваемый момент времени.

Применим изложенную выше методику к оценке надёжности единичного стержня при $\sigma_{0}(t)=\sigma_{0}=$ const, деформация которого описывается моделью $(1),(2)$ и задаётся соотношением (9). Для оценки надёжности необходимо знать математическое ожидание и дисперсию величины $p=p(t)$, которые находятся с использованием разложения (9). Тогда для математического ожидания имеем

$$
\mathrm{M}[p]=A_{1} \mathrm{M}[c]+A_{2} \mathrm{M}\left[c^{2} \alpha\right]-A_{3} \mathrm{M}\left[c^{3} \alpha^{2}\right]+A_{4} \mathrm{M}\left[c^{4} \alpha^{3}\right]
$$

а дисперсия деформации $p(t)$ определяется по классической формуле

$$
\begin{aligned}
\mathrm{S}_{p}^{2}=A_{1}^{2} \mathrm{~S}_{c}^{2}+ & A_{2}^{2} \mathrm{~S}_{c^{2} \alpha}^{2}+A_{3}^{2} \mathrm{~S}_{c^{3} \alpha^{2}}^{2}+A_{4}^{2} \mathrm{~S}_{c^{4} \alpha^{3}}^{2}+A_{1} A_{4} \mathrm{~K}_{c, c^{4} \alpha^{3}}+ \\
+2\left[A_{1} A_{2} \mathrm{~K}_{c, c^{2} \alpha}+A_{1} A_{3} \mathrm{~K}_{c, c^{3} \alpha^{2}}\right. & +A_{2} A_{3} \mathrm{~K}_{c^{2} \alpha, c^{3} \alpha^{2}}+ \\
& +A_{2} A_{4} \mathrm{~K}_{c^{2} \alpha, c^{4} \alpha^{3}}+A_{3} A_{4} \mathrm{~K}_{\left.c^{3} \alpha^{2}, c^{4} \alpha^{3}\right]}
\end{aligned}
$$

Здесь $\mathrm{M}[\cdot]$ - оператор математического ожидания; $\mathrm{S}_{p}^{2}, \mathrm{~S}_{c}^{2}, \mathrm{~S}_{c^{2} \alpha}^{2}, \mathrm{~S}_{c^{3} \alpha^{2}}^{2}, \mathrm{~S}_{c^{4} \alpha^{3}}^{2}-$ дисперсии соответствующих параметров; $\mathrm{K}_{c, c^{2} \alpha}, \mathrm{K}_{c, c^{3} \alpha^{2}}, \mathrm{~K}_{c^{2} \alpha, c^{3} \alpha^{2}}, \mathrm{~K}_{c^{2} \alpha, c^{4} \alpha^{3}}$, $\mathrm{K}_{c^{3} \alpha^{2}, c^{4} \alpha^{3}}$ - корреляционные моменты; $A_{1}=\sigma_{0}^{n} t, A_{2}=0,911 n \sigma_{0}^{2 n+1} t^{2}, A_{3}=$ $=-1,906 n^{2} \sigma_{0}^{3 n+2} t^{3}, A_{4}=3,061 n^{3} \sigma_{0}^{4 n+3} t^{4}$.

Для реализации методики необходимо «наполнение» соответствующих формул реальными экспериментальными данными. Для апробации методики использовались экспериментальные данные [24] по ползучести при чистом растяжении для 21 образца из одной плавки нержавеющей стали 12Х18Н10Т при $T=85^{\circ} \mathrm{C}$. Образцы испытывались на ползучесть при чистом растяжении вплоть до разрушения. Номинальные напряжения $\sigma_{0}$ принимали значения 39,24, 49,05, 58,86 и 78,48 МПа. Результаты экспериментов представлены в табл. 1. Здесь $\dot{p}_{0}=\dot{p}(0+0)$ - начальная скорость установившейся ползучести, $t_{1}$ и $p_{1}$ - экспериментальные значения времени и деформации в момент разрушения. 
Таблица 1

Результаты эксперимента и результаты расчёта случайных величин $\boldsymbol{c}$ и $\boldsymbol{\alpha}$

\begin{tabular}{c|c|c|c|c|c|c|c}
\hline № п/п & № обр. & $\sigma_{0}$, МПа & $\dot{p}_{0}$, час $^{-1}$ & $t_{1}$, час & $p_{1}$ & $c \cdot 10^{9}$ & $\alpha$ \\
\hline 1 & 5 & & 0,00080 & 35,0 & 0,048 & 6,365 & 0,198 \\
2 & 11 & & 0,00081 & 40,0 & 0,085 & 6,435 & 0,223 \\
3 & 16 & \multirow{3}{*}{39,24} & 0,00080 & 47,0 & 0,152 & 6,365 & 0,208 \\
4 & 13 & & 0,00084 & 66,0 & 0,234 & 6,673 & 0,142 \\
5 & 30 & & 0,00084 & 67,0 & 0,110 & 6,673 & 0,111 \\
6 & 32 & & 0,00081 & 68,0 & 0,125 & 6,435 & 0,124 \\
\hline 7 & 24 & & 0,0023 & 18,0 & 0,080 & 8,947 & 0,119 \\
8 & 22 & & 0,0019 & 20,5 & 0,090 & 7,391 & 0,141 \\
9 & 23 & & 0,0019 & 21,5 & 0,110 & 7,391 & 0,143 \\
10 & 27 & \multirow{2}{*}{49,05} & 0,0019 & 22,5 & 0,093 & 7,391 & 0,125 \\
11 & 26 & & 0,0021 & 24,0 & 0,130 & 8,169 & 0,114 \\
12 & 29 & & 0,0017 & 28,0 & 0,120 & 6,613 & 0,120 \\
13 & 28 & & 0,0014 & 30,0 & 0,080 & 5,446 & 0,117 \\
\hline 14 & 15 & & 0,0037 & 6,7 & 0,065 & 8,031 & 0,194 \\
15 & 31 & & 0,0027 & 14,0 & 0,047 & 5,861 & 0,051 \\
16 & 17 & \multirow{2}{*}{58,86} & 0,0023 & 15,0 & 0,073 & 4,992 & 0,127 \\
17 & 7 & & 0,0023 & 16,0 & 0,050 & 4,992 & 0,069 \\
18 & 14 & & 0,0033 & 20,0 & 0,170 & 7,163 & 0,073 \\
19 & 21 & & 0,0023 & 20,5 & 0,090 & 4,992 & 0,087 \\
\hline 20 & 39 & \multirow{2}{*}{78,48} & 0,0110 & 6,0 & 0,130 & 9,510 & 0,048 \\
21 & 37 & & 0,0045 & 6,0 & 0,118 & 3,890 & 0,146 \\
\hline
\end{tabular}

Значения $c$ и $\alpha$ находятся по результатам экспериментов для каждого образца (каждой реализации) при $n=3,2$ (определено в [24]), при этом величина $c$ определяется из соотношения

$$
c=\dot{p}_{0} / \sigma_{0}^{n}
$$

После определения величины $c$ значение $\alpha$ для каждой кривой ползучести определяется из условия прохождения графика через точку разрушения $\left(p_{1}, t_{1}\right)$, т. е. решения уравнения (3) относительно $\alpha$ при известных $p\left(t_{1}\right)=p_{1}, t=t_{1}$, $c, \sigma_{0}, n$. Результаты расчётов $c$ и $\alpha$ представлены в табл. 1.

Поскольку выборки случайных величин $c$ и $\alpha$ известны, не составляет труда найти выборки случайных величин $c^{2} \alpha, c^{3} \alpha^{2}, c^{4} \alpha^{3}$, а затем математические ожидания, дисперсии и коэффициент корреляции случайных величин $c, \alpha, c^{2} \alpha, c^{3} \alpha^{2}, c^{4} \alpha^{3}$. Результаты вычислений представлены в табл. 2 и 3. Далее с использованием полученных данных конкретизируются функции для математического ожидания и дисперсии (формулы (9) и (11) соответственно).

При известных математическом ожидании и дисперсии вычисляется ве-

Таблица 2

Математические ожидания и дисперсии случайных величин

\begin{tabular}{l|c|c|c|c|c}
\hline Случайная величина & $c$ & $\alpha$ & $c^{2} \alpha$ & $c^{3} \alpha^{2}$ & $c^{4} \alpha^{3}$ \\
\hline Математическое ожидание & $6,65 \cdot 10^{-9}$ & 0,128 & $5,80 \cdot 10^{-18}$ & $5,86 \cdot 10^{-27}$ & $6,58 \cdot 10^{-36}$ \\
\hline Дисперсия & $1,80 \cdot 10^{-18}$ & 0,002 & $8,28 \cdot 10^{-36}$ & $2,32 \cdot 10^{-53}$ & $5,52 \cdot 10^{-71}$ \\
\hline
\end{tabular}


роятность безотказной работы $P(t)$, при этом величина критической (допустимой) деформации $p^{*}$ в модельных расчётах полагалась 0,05:

$$
P(t)=\frac{1}{\sqrt{2 \pi} \mathrm{S}_{p(t)}} \int_{0}^{0,05} \exp \left(\frac{-(x-\mathrm{M}[p(t)])^{2}}{2 \mathrm{~S}_{p(t)}^{2}}\right) d x
$$

Таблица 3

Корреляционная матрица случайных ве-

\begin{tabular}{|c|c|c|c|c|}
\hline & $c$ & $c^{2} \alpha$ & $c^{3} \alpha^{2}$ & $c^{4} \alpha^{3}$ \\
\hline$c$ & 1 & 0,618 & 0,455 & 0,367 \\
\hline$c^{2} \alpha$ & & 1 & 0,966 & 0,908 \\
\hline$c^{3} \alpha^{2}$ & & & 1 & 0,984 \\
\hline$c^{4} \alpha^{3}$ & & & & 1 \\
\hline
\end{tabular}

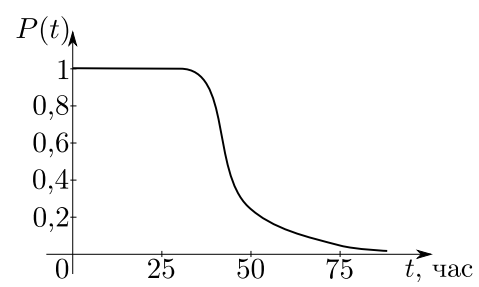

Вероятность безотказной работы $P(t)$

На рисунке в качестве примера приведена функция $P(t)$, полученная при $\sigma_{0}=39,24$ МПа. В табл. 4 приведены расчётные $t_{\text {расч }}$ и эксперимен-

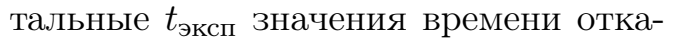
за с вероятностями 0,9, 0,95 и 0,99 для каждой реализации. При этом значе-

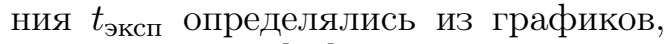
приведённых в [24].

Как следует из анализа данных табл. 4, при уровне вероятности 0,99 все экспериментальные значения (правый столбец таблицы) лежат правее времени безотказной работы, вычисленного по формуле (12). При значениях вероятности 0,95 и 0,9 имеются экспериментальные значения времени безотказной работы, которые лежат левее расчётного значения, т. е. имеются «выбросы» из расчётного значения ресурса. Поэтому в прикладных расчётах рекомендуется использовать величину вероятности 0,99.

\section{Таблица 4}

\section{Примеры расчёта вероятности безотказной работы}

\begin{tabular}{c|l|c|c}
\hline$\sigma_{0}$, МПа & $P(t)$ & $t_{\text {расч }}$, час & $t_{\text {эксп }}$, час \\
\hline \multirow{3}{*}{39,24} & 0,99 & 32 & 32,$25 ; 32,90$ \\
& 0,95 & 35 & 33,$5 ; 34,84$ \\
& 0,9 & 37 & 36,$13 ; 39,03$ \\
\hline \multirow{3}{*}{49,05} & 0,99 & 12,5 & 14,$35 ; 16,12$ \\
& 0,95 & 13,8 & 16,$3 ; 16,45$ \\
& 0,9 & 14,5 & 17,$42 ; 22,9$ \\
\hline \multirow{3}{*}{78,48} & 0,99 & 1,8 & \\
& 0,95 & 1,9 & 3,$67 ; 4,54$ \\
& 0,9 & 2 & \\
\hline
\end{tabular}

Таким образом, разработанный подход позволяет аналитическими методами прогнозировать величину назначенного ресурса для стержня по деформационному критерию отказа.

\section{БИБЛИОГРАФИЧЕСКИЙ СПИСОК}

1. Работнов Ю.Н. Ползучесть элементов конструкций. М.: Физматгиз, 1966.752 с. [Rabotnov Yu. N. Creep of Structural Elements. Moscow: Fizmatgiz, 1966. 752 pp.] 
2. Болотин В. В. Прогнозирование ресурса машин и конструкций. М.: Машиностроение, 1984. 312 с.; англ. пер.: Bolotin V. V. Prediction of service life for machines and structures. New York: ASME Press, 1989. 395 pp.

3. Ломакин B. А. Статистические задачи механики твердых деформируемых тел. М.: Наука, 1970. 139 с. [Lomakin V. A. Statistical Problems of the Mechanics of Solid Deformable Bodies. Moscow: Nauka, 1970. 139 pp.]

4. Самарин Ю. П. О применении стохастических уравнений в теории ползучести материалов // Изв. АН СССР. MTT, 1974. № 1. С. 88-94. [Samarin Yu.P. Use of stochastic equations in the theory of creep of materials // Izv. AN. SSSR. MTT, 1974. no. 1. Pp. 88-94].

5. Термопрочность деталей машин / ред. И. А. Биргер, Б. Ф. Шорр. М.: Машиностроение, 1975. 456 c. [ Thermal Resistance of Machine Parts / eds. I. A. Birger, B. F. Shorr. Moscow: Mashinostroenie, 1975. 456 pp.]

6. Бадаев А.Н. K вопросу об определении функции распределения параметров уравнения состояния ползучести // Пробл. прочности, 1984. №12. С. 22-26; англ. пер.: Badaev A.N. Determination of the distribution function of the parameters of a creep equation of state // Strength of Materials, 1984. Vol. 16, no. 12. Pp. 1668-1673.

7. Локощенко A.M., Шестериков С.А. Методика описания ползучести и длительной прочности при чистом растяжении // Ж. прикл. механики и технич. физики, 1980. № 3. C. 155-159; англ. пер.: Lokoshchenko A. M., Shesterikov S. A. Method for description of creep and long-term strength with pure elongation // J. Appl. Mech. Tech. Phys., 1980. Vol. 21, no. 3. Pp. 414-417.

8. Вилъдеман В.Э., Соколкин Ю.В., Ташкинов А.А. Механика неупругого деформирования и разрушения композиционных материалов. М.: Наука, 1997. 228 с. [Vil'deman V.É., Sokolkin Yu. V., Tashkinov A. A. Mechanics of inelastic deformation and fracture of composite materials. Moscow: Nauka, 1997. 228 pp.]

9. Сараев Л.А. Моделирование макроскопических пластических свойств многокомпонентных композиционных материалов. Самара: Самарский университет, 2000. 181 с. [Saraev L. A. Modelling of macroscopic plastic properties of multicomponent composite materials. Samara: Samarskiy Universitet, 2000. 181 pp.]

10. Самарин Ю. П. Стохастические механические характеристики и надежность конструкций с реологическими свойствами / В сб.: Ползучесть и длительная прочность конструкиий. Куйбышев: КПтИ, 1986. С. 8-17. [Samarin Yu.P. Stochastic Mechanical Properties and Reliability of Structures with Rheological Properties / In: Creep and LongTerm Strength of Structures. Kuibyshev: KPtI, 1986. Pp. 8-17].

11. Радченко В.П., Попов Н.Н. Стохастические характеристики полей напряжений и деформаций при установившейся ползучести стохастически неоднородной плоскости // Изв. вузов. Машиностроение, 2006. № 2. С. 3-11. [Radchenko V.P., Popov N. N. Stochastic characteristics of stress and strain fields in steady-state creep of stochastically inhomogeneous plane // Izv. Vuzov. Mashinostroenie, 2006. no. 2. Pp. 3-11].

12. Коваленко Л. В., Попов Н. Н., Радченко В. П. Решение плоской стохастической краевой задачи ползучести // ПMM, 2009. Т. 73, №6. С. 1009-1016; англ. пер.: Kovalenko L. V., Popov N.N., Radchenko V.P. Solution of the plane stochastic creep boundary value problem // J. Appl. Math. Mech., 2009. Vol.73, no. 6. Pp. 727-733.

13. Исуткина В. Н. Разработка аналитических методов решения стохастических краевых задач установившейся ползучести для плоского деформированного состояния: Автоpeф. дисс. ... канд. физ.-мат. наук. Самара, 2007. 18 с. [Isutkina V. N. Development of analytical methods for solving stochastic boundary value problems of steady-state creep for a flat strain state: Abstract of Ph. D. Thesis (Phys. \& Math.). Samara, 2007. 18 pp.]

14. Попов Н. Н., Радченко В. П. Нелинейная стохастическая задача ползучести неоднородной плоскости с учётом повреждённости материала // ПМТФ, 2007. Т. 48, № 2. С. 140146; англ. пер.: Popov N. N., Radchenko V.P. Nonlinear stochastic creep problem for an inhomogeneous plane with the damage to the material taken into account // J. Appl. Mech. Tech. Phys., 2007. Vol. 48, no. 2. Pp. 265-270. 
15. Бадаев А. Н., Голубовкий Е. Р., Баумштейн М. В., Бульцин И. П. О статистическом моделировании характеристик ползучести конструкционных материалов // Пробл. прочности, 1982. № 5. C. 16-20; англ. пер.: Badaev A. N., Gelubovskii E. R., Baumshtein M. V., Bulygin I. P. Statistical modeling of the creep characteristics of structural materials // Strength of Materials, 1982. Vol. 14, no. 5. Pp. 584-589.

16. Бадаев A.H. K вопросу об определении функции распределения параметров уравнения состояния ползучести // Пробл. прочности, 1984. №12. С. 22-26; англ. пер.: Badaev A. N. Determination of the distribution function of the parameters of a creep equation of state // Strength of Materials, 1984. Vol.16, no. 12. Pp. 1668-1673.

17. Ковпак В. И., Бадаев А. Н. Унифицированный подход к прогнозированию ползучести. Вопросы жаропрочных материалов в статистическом аспекте / В сб.: Унифицированнъе методы определения ползучести и длительной прочности. М.: Изд-во стандартов, 1986. C. 51-62. [Kovpak V.I., Badaev A. N. A Unified Approach to the Prediction of Creep. Issues of Heat-Resistant Materials in the Statistical Aspect/ In: Unified Methods of Determining Creep and Creep-Rupture Strength. Moscow: Izd-vo Standartov, 1986. Pp. 51$62]$.

18. Радченко В. П. Прогнозирование ползучести и длительной прочности материалов на основе энергетического подхода в стохастической постановке // Пробл. прочности, 1992. № 2. C. 34-40; англ. пер.: Radchenko V. P. Prediction of creep and creep-rupture strength of materials on the basis of an energy approach in a stochastic formulation // Strength of Materials, 1992. Vol. 24, no. 2. Pp. 153-161.

19. Исуткина В.Н., Маргаритов А. Ю. Сравнительный анализ решений стохастической краевой задачи установившейся ползучести для толстостенной трубы на основе методов малого параметра и Монте-Карло // Вестн. Сам. гос. техн. ун-та. Сер. Физ.мат. науки, 2006. № 43. C. 116-123. [Isutkina V. N., Margaritov A. Yu. A comparative analysis of solutions for stochastic boundary-value problem of the steady-state creep for thick-walled pipes on the basis of the small-parameter method and Monte Carlo simulation // Vestn. Samar. Gos. Tekhn. Univ. Ser. Fiz.-Mat. Nauki, 2006. no.43. Pp. 116-123].

20. Самарин Ю. П., Павлова Г. А., Попов Н. Н. Оценка надёжности стержневых конструкций по критерию деформационного типа// Пробл. машиностр. и надёжн. машин, 1990. № 4. C. 53-60. [Samarin Yu.P., Pavlova G. A., Popov N. N. Reliability estimation of beam structures by criterion of deformation type// Probl. Mashinostr. Nadezhn. Mash., 1990. no. 4. Pp. 53-60].

21. Попов Н.Н., Павлова Г.А., Шершнёва М.В. Оценка надёжности стержневых элементов конструкций из стохастически неоднородного разупрочнённого материала в условиях ползучести на основе параметрического критерия отказа // Вестн. Сам. гос. техн. ун-та. Сер. Физ.-мат. науки, 2010. №5(21). С. 117-124. [Popov N. N., Pavlova G.A., Shershneva M. V. Reliability estimation of stochastic heterogeneous rod constructional elements by the use of parametric failure criterion// Vestn. Samar. Gos. Tekhn. Univ. Ser. Fiz.-Mat. Nauki, 2010. no. 5(21). Pp. 117-124].

22. Радченко В.П., Ерёмин Ю.А. Реологическое деформирование и разрушение материалов и элементов конструкций. М.: Машиностроение-1, 2004. 264 с. [Radchenko V. P., Eremin Yu. A. Rheological Deformation and Fracture of Materials and Structural Members. Moscow: Mashinostroenie-1, 2004. 264 pp.]

23. Радченко В.П., Кичаев П.Е. Энергетическая концепция ползучести и виброползучести металлов. Самара: СамГТУ, 2011. 157 с. [Radchenko V. P., Kichaev P.E. Energetic concept of creep and cyclic strain-induced creep of metals. Samara: SamGTU, 2011. 157 pp.]

24. Соснин О.В., Никитенко А. Ф., Горев Б. В. Определение параметров кривых ползучести при наличии всех стадий процесса ползучести / В сб.: Расчёты и испытания на прочность. Расчетные методы определения несущей способности и долговечности элементов машин и конструкций. Метод определения параметров кривых ползучести и накопления повреждений при одноосном нагружении: Метод. рекомендации. М.: ВНИИНМАШ, 1982. С. 49-54. [Sosnin O.V., Nikitenko A.F., Gorev B. V. Definition of creep curves parameters by the presence of all stages of creep process / In: Calculations and 
tests of strength. Calculated methods for determining the bearing capacity and durability of machine elements and structures. The method of creep curves parameters determination and the accumulation of damage under uniaxial loading: Methodological Specifications. Moscow: VNIINMASh, 1982. Pp. 49-54].

Поступила в редакцию 20/XI/2011;

в окончательном варианте - 27/I/2012.

MSC: 74S60; 74K10, 60H30

\section{CALCULATION METHOD FOR FRAME CONSTRUCTION LIFE PREDICTION ON THE BASIS OF CREEP AND ENDURANCE OF ENERGY TYPE}

\section{V. Shershneva}

Samara State Technical University, 244, Molodogvardeyskaya st., Samara, 443100, Russia.

E-mail: mary-sofya@mail.ru

The analytical method of estimation of frame construction life prediction in terms of creep by strain failure criterion is suggested. The linearization of stochastic rheological energy equations, is made, the estimations of uptime under the assumptions on the limit values of creep are obtained. The checking of accordance of the method with the experimental data for the creep of samples made of $12 \mathrm{Kh} 18 \mathrm{~N} 10 \mathrm{~T}$ steel under $\mathrm{T}=850{ }^{\circ} \mathrm{C}$ is implemented. There is agreement between the calculated and experimental data.

Key words: creep, stress-rupture strength, stochastic model, probability of no-failure, strain failure criterion.

Original article submitted 20/XI/2011;

revision submitted $27 / \mathrm{I} / 2012$.

Mariya V. Shershneva, Postgraduate Student, Dept. of Applied Mathematics \& Computer Science. 